\title{
Exploring Extra Dimensions in Spectroscopy Experiments
}

\author{
Feng Luo* and Hongya Liu ${ }^{\dagger}$ \\ Department of Physics, Dalian University of Technology, Dalian, 116024, P. R. China
}

\begin{abstract}
We propose an idea in spectroscopy to search for extra spatial dimensions as well as to detect the possible deviation from Newton's inverse-square law at small scale, and we take high-Z hydrogenic systems and muonic atoms as illustrations. The relevant experiments might help to explore more than two extra dimensions scenario in ADD's brane world model and to set constraints for fundamental parameters such as the size of extra dimensions.
\end{abstract}

PACS numbers: 04.80.-y, 11.10.Kk, 32.30.-r, 36.10.-k

Keywords: Precision spectroscopy; Extra dimensions; Newton's inverse-square law.

\footnotetext{
*fluo@student.dlut.edu.cn

† hyliu@dlut.edu.cn
} 


\section{INTRODUCTION}

The possibility that there exists more than three spatial dimensions in nature has aroused physicists' interests for many years. Recently, the studying focus of theories about extra dimensions has shifted to the "brane world" scenario. In contrast to the original string/superstring theories in which extra dimensions are assumed to be as small as Planck scale $\left(\sim 10^{-33} \mathrm{~cm}\right)$ and hence the detection of these tiny dimensions is hopeless, extra dimensions in brane world scenario can be large or even infinite (for a review, see e.g., 1] ), so that the search for such hidden dimensions becomes much more encouraging.

Among various brane world models, the one proposed by ADD (N. Arkani-Hamed, S. Dimopoulos and G. Dvali) is especially interesting [2], 3], 4], and its implications in accelerator physics, astrophysics and cosmology have been widely studied (see, for example, [5], 6] , 7] ). Perhaps the most distinctive character of this model is it predicts that the familiar Newton's inverse-square law (ISL) would fail below the size of extra dimensions. The reason is as follows: to solve the hierarchy problem in particle physics, that is, the unnatural huge energy gap between Planck mass $M_{p l} \sim 10^{19} \mathrm{Gev}$ and electroweak mass $m_{E W} \sim 10^{3} \mathrm{Gev}$, ADD assume the four dimensional $M_{p l}$ is not a fundamental scale, but an induced one from the $(4+n)$ dimensional Planck mass $M_{p l(4+n)}$ through

$$
M_{p l}^{2} \sim M_{p l(4+n)}^{2+n} R^{n},(c=1, \hbar=1)
$$

where $n$ and $R$ are the number and radius of the extra dimensions respectively. For $M_{p l(4+n)}=1 T e v$, this equation is equivalent to

$$
R \sim 10^{-17+\frac{32}{n}} \mathrm{~cm}
$$

They further assume $M_{p l(4+n)}$ is around the scale of $m_{E W}$, then the hierarchy becomes trivial. Considering within brane world scenario gravitational field (with possible exception of some hypothetic very weakly coupled fields) is the only field that can propagate in extra dimensions, a straightforward use of $(4+n)$ dimensional Gauss' law infers that the usual ISL of gravitational force would change to a much stronger one at small scale as

$$
F \propto \frac{1}{r^{n+2}}, \text { for } r \ll R,
$$

and it recovers to ordinary ISL for $r \gg R$. While the $n=1$ case, for which $R \sim 10^{12} m$ from Eq.(2), is excluded from planetary motion observations, other possibilities of $n$ cannot be 
boldly ruled out. Particularly, the $n=2$ case implies sub-millimeter extra dimensions, while the experimental conditions for testing Newton's ISL by torsion pendulum method was just about to be available when ADD's proposal appeared, therefore, many people have devoted to the search of deviation from ISL as well as extra dimensions during the past few years [8], 9], [10], 11]. By now, there is no reported deviation from ISL and the parameter $M_{p l(4+n)}$ has been constrained to larger than several Tev by torsion-balance experiments ([11], [6], including also constraints from accelerators and astrophysics etc.).

Notice that Eq.(2) results in smaller $R$ for larger $n$. For $n=3$, one obtains $R \sim 10^{-7} \mathrm{~cm}$. Direct measurement of gravitational force using torsion pendulum at such small scale is far beyond the reach of current and foreseeable future experimental ability. (Note, however, that the scales of different extra dimensions are not guaranteed to be the same, so the deviation from ISL may also appear in micron range even for $n>2$.) Considering the limitation of torsion-balance experiments and the importance of detection of the possible deviation from ISL as well as exploring extra dimensions, searching for other alternative experimental methods is worthwhile. It is well know that spectroscopy is one of the most precise and thoroughly studied fields in physics, so we wonder whether clues about deviation from ISL and extra dimensions can be found in such field. The idea is quite simple: since gravity may become much stronger at small scale, then for small scale systems, such as atoms, ions and even subatoms, effects of the original safely neglected gravity may actually be large enough and able to show itself in spectroscopic spectra. Because of the precision of spectroscopy, such experiments may help to set constraints of $M_{p l(4+n)}$ and $R$, or some other model depended parameters. Moreover, these spectroscopic experiments may be capable of dealing with the $n=3$ case and detect the possible deviation from ISL down to nanometer range.

In a previous paper [12], we did some calculations based on one-proton one-lepton systems, that is, hydrogen atom, muonic hydrogen etc., and found that the corrections from the strengthened gravity for the ground state binding energy of these systems are many orders of magnitude larger than the ones from the exact ISL gravity. Now we would like further develop our work to high-Z hydrogenic systems (one electron) and muonic atoms, which are particular interesting in precision spectroscopy since their important role in testing quantum electrodynamics (QED) (see, for example, [13], [14], [15], 16]). The effects of gravity in such systems are much larger and hence may be more encouraging for our proposal. In this sense, 
these systems may be not only suitable to test the Standard Model of particle physics, but also help to explore scenarios beyond the Standard Model.

\section{GRAVITY CORRECTIONS IN HIGH-Z HYDROGENIC SYSTEMS}

Even within ADD's framework where gravity becomes much stronger at small scale, gravity is still far smaller compared to electromagnetic force in high-Z hydrogenic systems, so we can conveniently treat it as a perturbation. Also, for estimating gravity effects from the view of orders of magnitude, we just need to consider corrections from the leading Schrodinger term.

As an illustration, we will only perform calculations for the ground state energy level, which is also the simplest case. Clearly, correction in this energy level is the largest one because of the smallest Bohr radius, which serves as the mean distance between the gravitational sources - the atomic nucleus and the electron.

The first-order correction of the ground state energy level is written as

$$
\Delta E=\int_{0}^{\infty} \int_{0}^{2 \pi} \int_{0}^{\pi} \Psi_{100}^{*} \hat{V}(r) \Psi_{100} r^{2} \sin \theta d \theta d \phi d r
$$

where $\Psi_{100}=\pi^{-\frac{1}{2}} a^{-\frac{3}{2}} e^{-\frac{r}{a}}$ is the ground state wave function of the high-Z hydrogenic system, $a=\frac{\hbar^{2}}{m e^{2} Z}$ is the first Bohr radius, $m=\frac{m_{l} M}{m_{l}+M}$ is the reduced mass, $m_{l}$ and $M$ are the masses of electron and atomic nucleus respectively. The gravitational potential is given as

$$
\hat{V}(r)=\left\{\begin{array}{cl}
-\frac{G_{(4+n)} m_{l} M}{r^{n+1}}, & r \ll R \\
-\frac{G_{4} m_{l} M}{r}\left(1+\alpha e^{-\frac{r}{\lambda}}\right), & r \sim R, \\
-\frac{G_{4} m_{l} M}{r}, & r \gg R
\end{array}\right.
$$

where the $(4+n)$ dimensional Newton's constant is $G_{(4+n)} \sim R^{n} G_{4}$, and the four dimensional Newton's constant is $G_{4}=M_{p l}^{-2}$. The second line of Eq.(5) takes the Yukawa type, in which the parameters $\alpha \sim n$ and $\lambda \sim R$. In above expressions, we have neglected constants of order unity which depends on the specific compactification forms of extra dimensions, and we have assumed that the sizes of all the extra dimensions are the same. More detail expressions can be seen in [10], [12].

From Eq.(4) and the first line of Eq.(15), one can notice that the integral diverges as $r \rightarrow 0$ for $n \geq 2$. Considering the atomic nucleus is not point like, we introduces a safe cutoff value 
$r_{m}$ with the atomic nucleus size for the lower limit of the integral. A convenient expression of $r_{m}$ is $r_{m}=r_{0} A^{\frac{1}{3}}$, where $A$ is the mass number of the atomic nucleus and $r_{0}$ is of size $\sim 10^{-13} \mathrm{~cm}$. Also note that such cutoff leads to a relatively conservative estimation of the gravity effects.

Neglecting coefficients of order unity, the above equations give

$$
\Delta E \sim\left\{\begin{array}{cc}
-G_{4} m_{l} M a^{-1}, & n=0 \\
-M_{p l(4+n)}^{-3} m_{l} M a^{-2}, & n=1 \\
-M_{p l(4+n)}^{-4} m_{l} M a^{-3}, & n=2 . \\
-M_{p l(4+n)}^{-5} m_{l} M a^{-3} r_{m}^{-1}, & n=3 \\
-M_{p l(4+n)}^{-6} m_{l} M a^{-3} r_{m}^{-2}, & n=4
\end{array}\right.
$$

Since $r_{m} \sim 10^{-12} \mathrm{~cm}$ and $M_{p l(4+n)}^{-1} \sim 10^{-17} \mathrm{~cm}$ for $M_{p l(4+n)} \sim 1 T e v$, then for $n \geq 4$, the corrections are much smaller than the ones for $n=2$ and $n=3$, which are the cases of our interest.

In fact, for $n=2$ and $n=3, \Delta E \propto A Z^{3}$ and $\Delta E \propto A^{\frac{2}{3}} Z^{3}$ respectively. Therefore, it is clear that the corrections for high- $Z$ hydrogenic systems are much larger compared to the ones for hydrogen atom. Convert $\Delta E$ to corrections in terms of frequency $\Delta \nu$ through $\Delta E=h \Delta \nu$. For a specific system, say ${ }^{207} \mathrm{~Pb}^{81+}, \Delta \nu \sim 10^{0} \mathrm{~Hz}$ for $n=2$ and $10^{-5} \mathrm{~Hz}$ for $n=3$, while the corresponding corrections in hydrogen atom are $10^{-8} \mathrm{~Hz}$ and $10^{-13} \mathrm{~Hz}$ respectively. Also note that the exact ISL, that is, the $n=0$ case gives a correction as small as $\Delta \nu \sim 10^{-24} \mathrm{~Hz}$ for hydrogen atom.

The above calculations also suit to muonic atoms (one muon in the atoms or ions, not confined to hydrogen-like), since the ground state muonic orbit is far interior compare to the first electronic Bohr orbit and hence the muon sees approximately a pure Coulomb potential from the atomic nucleus. In this case, what we need to do is just assigning $m_{l}$ in Eq.(6) the mass of muon, which is about 200 times larger than electron. Notice $m_{l}$ also appears in the expression of $a$, such substitution further results in several orders of magnitude increase of $\Delta \nu$. For ${ }^{207} \mathrm{~Pb}$ muonic atom, $\Delta \nu \sim 10^{9} \mathrm{~Hz}$ for $n=2$ and $10^{4} \mathrm{~Hz}$ for $n=3$. For a medium-Z muonic atom, e.g., ${ }^{40} \mathrm{Ca}$ muonic atom, $\Delta \nu \sim 10^{6} \mathrm{~Hz}$ for $n=2$ and $10^{2} \mathrm{~Hz}$ for $n=3$.

The sensitivity of $\Delta \nu$ to $M_{p l(4+n)}$ and the corresponding $R$ (notice Eq.(11)) is easily seen from Eq.(6), since $\Delta \nu \propto M_{p l(4+n)}^{-4}$ and $\Delta \nu \propto M_{p l(4+n)}^{-5}$ for $n=2$ and $n=3$ respectively. Fig. 1 1 show the ${ }^{40} \mathrm{Ca}$ muonic atom case for $n=2$, and Fig. 2 show the ${ }^{207} \mathrm{~Pb}$ muonic atom case for $n=3$. 


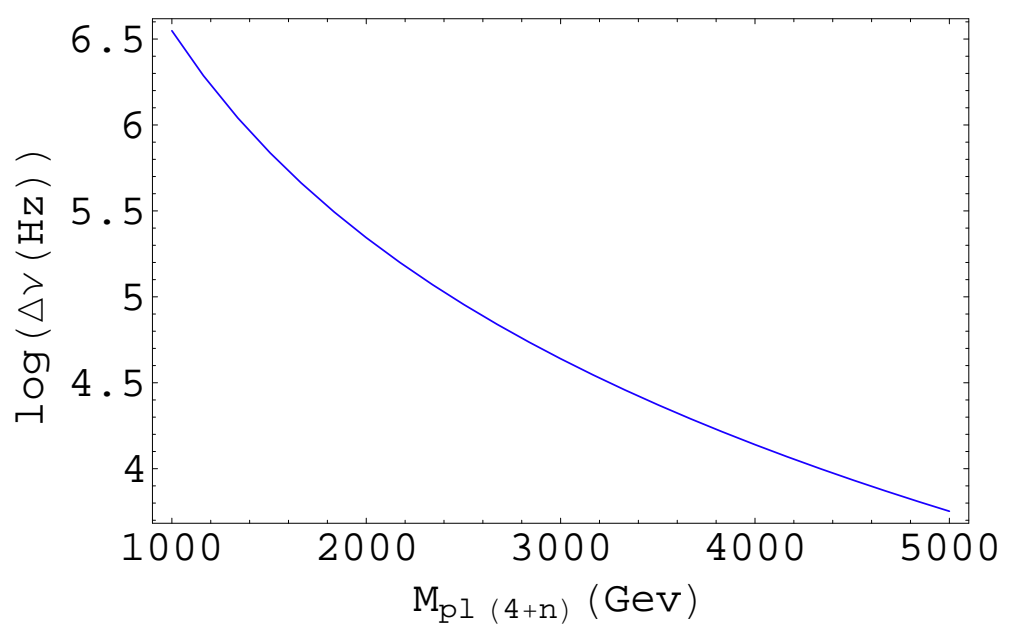

FIG. 1: Dependence of $\Delta \nu$ to $M_{p l(4+n)}$ for ${ }^{40}$ Ca muonic atom, $n=2$.

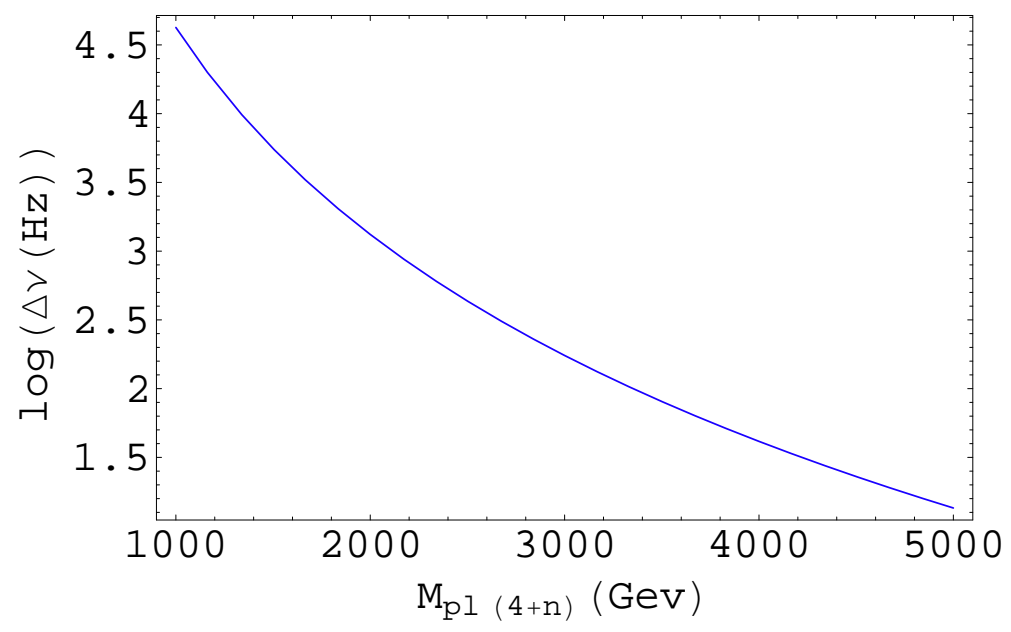

FIG. 2: Dependence of $\Delta \nu$ to $M_{p l(4+n)}$ for ${ }^{207} \mathrm{~Pb}$ muonic atom, $n=3$.

\section{CONCLUSIONS AND DISCUSSIONS}

Considering the above large corrections from gravitational effects, it appears spectroscopic experiments using high-Z hydrogenic systems and medium-Z or high-Z muonic atoms may serve as new methods to detect the possible deviation of Newton's ISL as well as to search for extra dimensions. The high precision X-ray and laser spectroscopy make this proposal interesting, since the relevant experiments may not only be able to explore the $n=2$ case in ADD's brane world model, but also make the study of $n=3$ case and nanometer range deviation possible. Also, the strong dependence of the corrections to fundamental parameters of the model, e.g., the scales of extra dimensions, may help to further constrain 
such parameters.

We note, however, great efforts may have to be made before the realization of our proposal. Theoretically, the uncertainties of the nuclear structure effects, the higher-order terms neglected in calculation of other effects etc., may submerge the effects of gravity. Experimentally, to achieve enough accuracy to show the strengthened gravity effects is also challenging (for a review about experimental accuracy for Lyman- $\alpha$ transitions in high-Z hydrogenic systems, see [17]).

Anyway, although difficulties exist, it is fair to say that in precision spectroscopy, similar ideas may find their way in many other systems, e.g., subatomic and sub-nuclear systems, and by various experiments, e.g., experiments involving hyperfine structures. In a word, precision spectroscopy may play an active role in the development of the most exotic theories of particle physics and cosmology, and it may really help to explore those most exotic mysteries like extra dimensions.

\section{ACKNOWLEDGEMENTS}

This work was supported by NSF (10573003) and NBRP (2003CB716300) of P. R. China.

[1] V. A. Rubakov, Phys. Usp. 44 (2001) 871-893; Usp. Fiz. Nauk 171 (2001) 913-938, hep-ph/0104152.

[2] N. Arkani-Hamed, S. Dimopoulos, G. Dvali, Phys. Lett. B429 (1998) 263, hep-ph/9803315.

[3] I. Antoniadis, N. Arkani-Hamed, S. Dimopoulos and G. Dvali, Phys. Lett. B436 (1998) 257, hep-ph/9804398.

[4] N. Arkani-Hamed, S. Dimopoulos, G. Dvali, Phys. Rev. D59 (1999) 086004, hep-ph/9807344.

[5] S. Hannestad, G. Raffelt, Phys. Rev. Lett. 87 (2001) 051301, hep-ph/0103201.

[6] P. Kanti, Int. J. Mod. Phys. A19 (2004) 4899, hep-ph/0402168.

[7] F. Luo, H. Liu, Int. J. Theor. Phys., Vol 44, No 9 (2005) 1441, gr-qc/0503115.

[8] J. C. Long, H. W. Chan, A. B. Churnside, E. A. Gulbis, M. C. M. Varney, J. C. Price, Nature 421 (2003) 922.

[9] J. C. Long, J. C. Price, Comptes Rendus Physique 4 (2003) 337, hep-ph/0303057. 
[10] E. G. Adelberger, B. R. Heckel, A. E. Nelson, Ann. Rev. Nucl. Part. Sci. 53 (2003) 77, hep-ph/0307284.

[11] C. D. Hoyle, D. J. Kapner, B. R. Heckel, E. G. Adelberger, J. H. Gundlach, U. Schmidt, H. E. Swanson, Phys. Rev. D70 (2004) 042004, hep-ph/0405262.

[12] F. Luo, H. Liu, to appear in Int. J. Theor. Phys., gr-qc/0602093.

[13] P. Beiersdorfer, S. B. Utter, K. L. Wong, J. R. Crespo Lopez-Urrutia, J. A. Britten, H. Chen, C. L. Harris, R. S. Thoe, D. B. Thorn, E. Trabert, Phys. Rev. A64, (2001) 032506.

[14] H. F. Beyer, G. Menzel, D. Liesen, A. Gallus, F. Bosch, R. D. Deslattes, P. Indelicato, Th. Stohlker, O. Klepper, R. Moshammer, F. Nolden, H. Eickhoff, B. Franzke, M. Steck, Z. Phys. D35, (1995) 169.

[15] I. Klaft, S. Borneis, T. Engel, B. Fricke, R. Grieser, G, Huber, T. Kuhl, D. Marx, R. Neumann, S. Schroder, P. Seelig, L. Volker, Phys. Rev. Lett. 73, (1994) 2425.

[16] L. S. Brown, R. N. Cahn, and L. D. McLerran, Phys. Rev. Lett. 32, (1974) 562.

[17] J. L. Flowers, H. A. Klein, D. J. E. Knight and H. S. Margolis, Hydrogenic Systems for Calculable Frequency Standards: Status and Options, NPL report CBTLM 11 (2001). 